\title{
Delayed-onset Necrotizing Myositis following COVID-19 Infection
}

\author{
Sravani Lokineni ${ }^{1}$, Mahta Mortezavi ${ }^{2}$ \\ ${ }^{1}$ Department of Internal Medicine, Rochester Regional Health, Rochester, NY, USA \\ ${ }^{2}$ Department of Allergy, Immunology and Rheumatology, Rochester Regional Health, Rochester, NY, USA
}

Received: 07/03/2021

Accepted: $11 / 03 / 2021$

Published: 20/04/2021

How to cite this article: Lokineni S, Mortezavi M. Delayed-onset necrotizing myositis following COVID-19 infection. EJCRIM 2021;8: doi:10.12890/2021_002461.

Conflicts of Interests: Dr Lokineni has nothing to disclose. Dr Mortezavi was in clinical practice when she saw and cared for this patient before she started working for Pfizer Global Medical Affairs-Xeljanz in October, 2020. This manuscript was not prepared with help from Pfizer

This article is licensed under a Commons Attribution Non-Commercial 4.0 License

\section{ABSTRACT}

As the numbers of cases of COVID-19 continue to rise, the heterogeneity of its clinical manifestation continues to increase. Here, we describe a case of delayed-onset, biopsy-proven necrotizing myositis following infection with SARS-CoV-2.

\section{LEARNING POINTS}

- SARS-CoV-2 may be associated with late-onset necrotizing myositis, mimicking autoimmune inflammatory myositis.

- We need more studies to understand the underlying pathogenesis of SARS-CoV-2- induced myositis.

\section{KEYWORDS}

COVID-19, SARS-CoV-2, necrotizing autoimmune myositis

\section{CASE DESCRIPTION}

Our patient was a 51-year-old Chilean-American man who had previously been in good health. He worked in manufacturing and had to climb up and down ladders and lift heavy boxes on a regular basis as part of his job. His medical history included osteoarthritis of the cervical spine and cervical fusion at the C4/C5 level. He also reported having an episode of alopecia areata about 15 years previously, which had resolved spontaneously. He did not have a history of respiratory disease and did not smoke.

At the end of April of 2020, he was exposed to SARS-CoV-2 at work. A few days later, he started to experience fever, fatigue and shortness of breath and was diagnosed with COVID-19 by nasal swab. By early May, his shortness of breath had progressed and he was admitted to the hospital with multifocal pneumonia and treated with broad-spectrum antibiotics and convalescent plasma, with seemingly good results. In late June, he developed fever and shortness of breath again, and since he continued to have positive tests, the question of a protracted course versus reinfection was raised. He was treated with dexamethasone and antibiotics during a second hospital admission and discharged home again.

Three months after the initial infection, the patient began to experience palpitations in addition to the ongoing shortness of breath. He presented again to the hospital, where he had a full evaluation, including a cardiac work-up, which revealed a slight troponin elevation to 0.19 $\mathrm{ng} / \mathrm{ml}$ and an elevated CK of $9600 \mathrm{U} / \mathrm{l}$. Over the following days, his CK continued to rise to 27,000 U/I and his physical exam demonstrated weakness of both the upper and lower extremities. He was fluid resuscitated for presumed rhabdomyolysis, but his CK did not improve. Rheumatology saw him at this point and started oral prednisone 60 mg daily for 3 weeks with improvement in his muscle weakness and a 
downward trend in his CK to 12,000 U/I. The autoimmune work-up included a myositis panel (Mi-2, Ku, PM-Scl100, PM-Scl75, Jo-1, SRP, PL-7, PL-12, EJ, OJ, Ro-52), anti-nuclear antibody (ANA), anti-double stranded-DNA (dsDNA), anti-Smith, anti-RNP, anti-SSA, anti-SSB, antineutrophilic cytoplasmic antibody (ANCA), myeloperoxidase and proteinase-3 antibody, all of which were negative. His CK level rose when his steroid dose was tapered below $20 \mathrm{mg}$ daily. Therefore, azathioprine $150 \mathrm{mg}$ daily $(2 \mathrm{mg} / \mathrm{kg}$ ) was added as a steroid-sparing agent to allow for further tapering. He was also referred for physical therapy, which the patient felt was helped with his weakness.

Photographs of the patient's muscle biopsy specimen are shown in Fig. 1. The specimen showed atrophic fibres in a perifascicular pattern, with frequent necrotic and basophilic fibres with moderate numbers of split fibres. Staining with trichrome, nucleotide adenine dinucleotide $(\mathrm{NADH})$ reaction, succinic dehydrogenase $(\mathrm{SDH})$ and cytochrome c oxidase (COX) did not reveal any abnormalities. These findings were suggestive of acute necrotizing myositis.
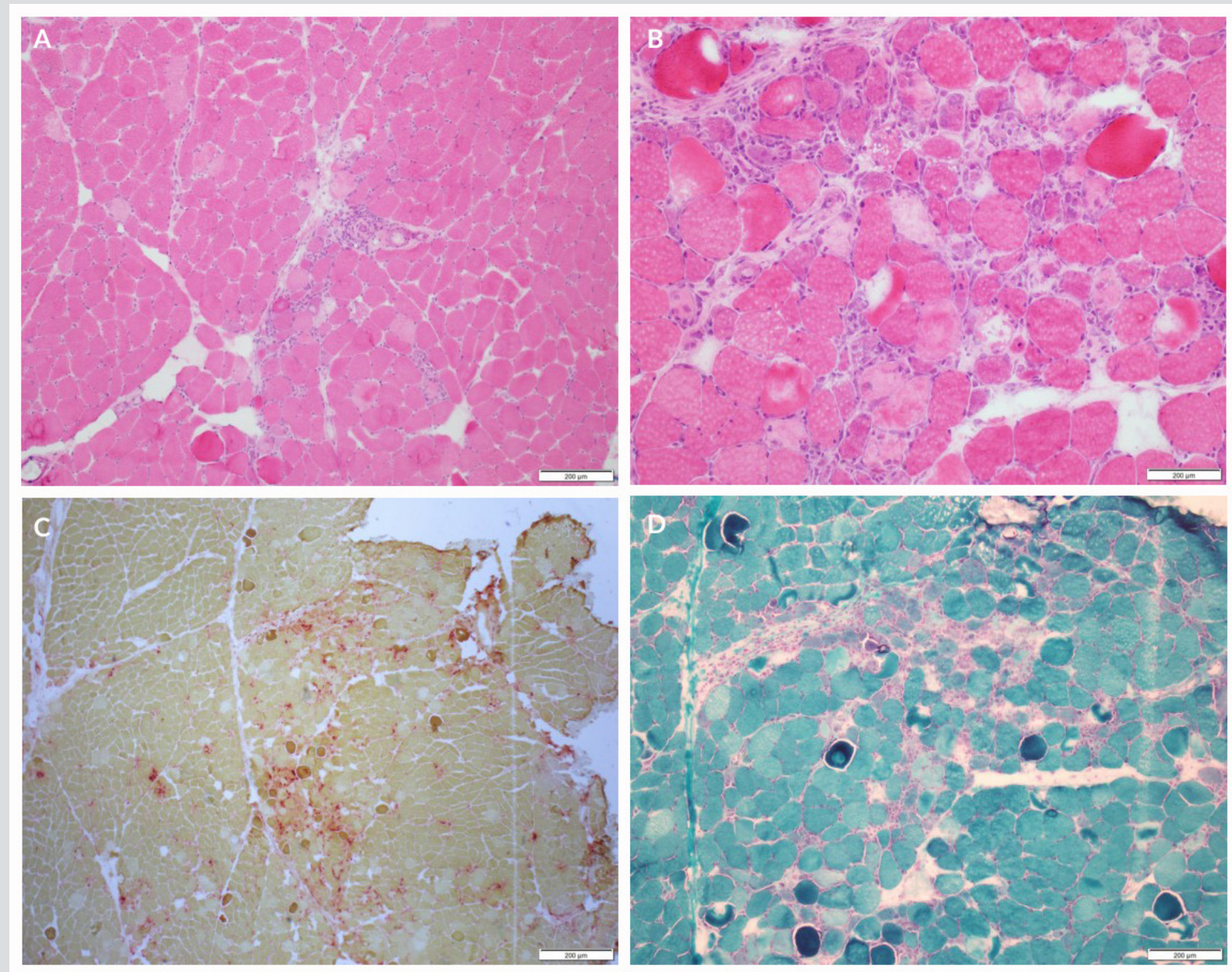

Figure 1. (A) H\&E which shows perimysial perivascular mononuclear inflammatory cell infiltrates at $10 \times$ magnification. (B) H\&E which shows necrotic and basophilic fibres at $40 \times$ magnification. (C) Gomori trichrome stain which shows necrotic and basophilic fibres at $10 \times$ magnification. (D) Acid phosphatase stain which shows the necrotic fibres staining red at $4 \times$ magnification; the necrotic fibres have faint pink cytoplasm. Images are courtesy of the Neuromuscular Laboratory at the University of Rochester

\section{DISCUSSION}

The underlying pathogenesis of SARS-CoV-2-induced muscle damage is not well understood. The possible proposed mechanisms of myositis in our patient included direct skeletal muscle damage by SARS-CoV-2 or immune-mediated muscle damage mediated by type I interferon ${ }^{[1]}$ leading to inflammation and necrosis of the muscles. Interestingly, necrotizing autoimmune myositis (NAM) has been reported with other viral infections including coxsackie B, parvovirus, enterovirus, human T cell-lymphotropic virus (HTLV-1) and human immunodeficiency virus $(\mathrm{HIV})^{[2,3]}$. NAM can present as an acute part of the initial infection or a subacute late-onset sequela. 


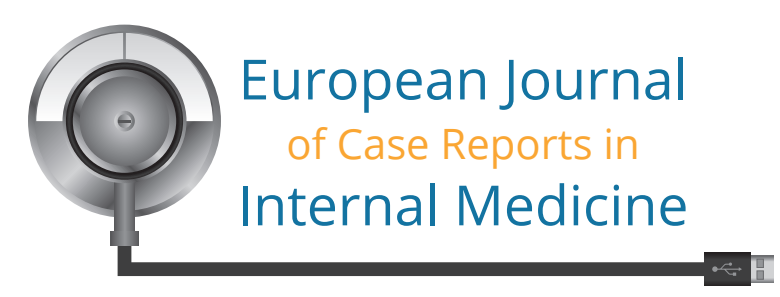

Although our patient had negative serologies, patients with NAM can have antibodies against signal recognition particle (SRP) or hydroxy3-methylglutaryl-coenzyme A reductase (HMGCR), but the pathogenic role of these antibodies is not clear ${ }^{[3]}$. Muscle biopsy is the gold standard for diagnosis and shows findings of necrotic and regenerative muscle fibres.

Up to $35.8 \%$ of patients with COVID-19 report myalgia during the acute phase of the infection ${ }^{[4]}$. There are reported cases of patients with COVID-19 developing rhabdomyolysis, critical illness myopathy, Guillain-Barre syndrome and neuropathy ${ }^{[4]}$. Three previous cases of acute COVID-19-induced myositis have been reported ${ }^{[5,6]}$. Our case highlights the already described protracted course of this viral infection in some patients and the potential for delayed onset necrotizing myositis necessitating long-term immunosuppression.

\section{REFERENCES}

Manzano GS, Woods JK, Amato AA. Covid-19-associated myopathy caused by type I interferonopathy. N Engl J Med 2020;383:2389-2390.

Dalakas MC. Inflammatory muscle diseases. N Engl J Med 2015;372(18):1734-1747.

Adler BL, Christopher-Stine L. Triggers of inflammatory myopathy: insights into pathogenesis. Discov Med 2018;25(136):75-83.

Paliwal VK, Garg RK, Gupta A, Tejan N. Neuromuscular presentations in patients with COVID-19. Neurol Sci 2020;41(11):3039-3056.

Zhang H, Charmchi Z, Seidman RJ, Anziska Y, Velayudhan V, Perk J. COVID-19-associated myositis with severe proximal and bulbar weakness. Muscle Nerve 2020;62(3):E57E60.

6. BeydonM,Chevalier K,AlTabaa O,HamrounS,DelettreAS,ThomasM, etal.MyositisasamanifestationofSARS-CoV-2.AnnRheumDis 2020Apr 23:annrheumdis-2020-217573. doi: 10.1136/annrheumdis-2020-217573. Online ahead of print. 